\title{
PHASE SWITCHING AND PHASE BALANCING TO COPE WITH A MASSIVE PHOTOVOLTAIC PENETRATION
}

\author{
Sam Weckx \\ KULeuven - Belgium \\ sam.weckx@esat.kuleuven.be
}

\author{
Carlos GonzalezDeMiguel \\ KULeuven - Belgium \\ carlos.gonzalezdemiguel@esat.kuleuven.be pieter.vingerhoets@esat.kuleuven.be \\ Johan Driesen \\ KULeuven - Belgium \\ johan.driesen@esat.kuleuven.be
}

\begin{abstract}
This paper compares two methods that result in a more balanced operation of the electricity grid. A balanced distribution grid results in improved voltage profiles, a lower voltage unbalance and lower losses. The first method discusses the changing of the phase connection of single-phase customers. Optimization techniques with a constrained maximum number of changes show that this passive solution may be suitable for unbalanced grids. Secondly the effect of balancing three-phase PV-inverters is investigated. The proposed control consists of injecting the majority of the produced power in the phase with the highest power consumption, based only on local voltage information. Both methods are evaluated with extensive load flow simulations with real data.
\end{abstract}

\section{INTRODUCTION}

In the recent years, many photovoltaic (PV) installations have been connected to the Low-Voltage (LV) distribution networks. Their introduction in the grid causes a reduction or reversal of the power flow, which results in a voltage increase in the LV network [1]. A high amount of PV generation might result as well in voltage unbalance [2]. Different approaches are proposed to compensate for voltage rise and voltage unbalance, including droop control on active power, power curtailment, reactive power compensation and negative sequence current droop control [3] [4] [5]. This paper evaluates two methods under less attention to cope with an increasing amount of PV.

The first mitigating solution proposed in this paper is a passive measure and consists of changing the phase where the customers are connected, in order to compensate or redistribute them in a more balanced way. The goal is to reduce the unbalance and to minimize the number of phase changes the DSO should do.

The other method investigated is the active balancing of the grid by power electronics. Three-phase PV inverters can be replaced by three separately controlled single phase inverters with common DC-bus. PV inverters rarely operate at maximal power production. This allows to inject the majority of produced power in the phase with the highest power consumption, without overloading one of the single phase inverters. The extra flexibility of three separate single phase inverters comes at the cost of requiring six inverter legs, instead of three or four and a harder DC-bus voltage control. However, the use of three separate single phase inverters is often already more interesting than one three-phase inverter, due to disconnection regulations [6]. In case voltage rises above a certain threshold, typically $+10 \%$, the inverter has to disconnect. In case three separate single phase inverters are used, only the inverter connected to the overloaded phase has to disconnect. This results in a higher amount of energy that can be delivered to the grid.

\section{PHASE SWITCHING}

The main purpose of the phase switching is to improve the distribution of the load across the three phases. In this section a method is proposed to suggest to the DSO which customers to switch to which phase.

The problem is constrained to a limited number of phase changes, since a significant reduction of the unbalance is achievable with only few changes. Moreover, the more the changes, the higher the cost for the DSO [7]. Different objectives can be taken to be minimized, like the minimization of the Voltage Unbalance Factor (VUF). To make the optimization problem tractable, the unbalance optimization is simplified and turned into the minimization of the phase voltage difference:

$$
\begin{aligned}
& \min _{P h_{h}} . \quad\left|V_{U}-V_{V}\right|+\left|V_{U}-V_{W}\right|+\left|V_{V}-V_{W}\right| \\
& \text { subject to } \quad \sum_{h=1}^{3} P h_{h}=1 \\
& \text { Loadflow }
\end{aligned}
$$

the vector $P h_{h}$ codifies the phase where the single-phase customer is connected to. For instance, $P h_{h}=[0,0,1]$ indicates that the load is connected to phase 3 , the only non-zero element. Loadflow denotes a set of power flow constraints which can be found in [8]. In addition, the problem is simplified by optimizing the unbalance only in the node that presented the highest unbalance level given the original phase configuration, reducing the required computation time, but resulting in a possible 
suboptimal solution when this node has not the highest unbalance after the optimization. The formulation of the problem includes the constraint of the maximum number of changes. Given an initial phase configuration $P h_{0}$ and the optimal configuration $P h^{*}$, the number of changes can be written as:

$$
n_{\text {changes }}=\sum<P h_{0} \cdot P h^{*}>
$$

In order to accelerate the nonlinear equations of the load flow calculations, the power flow constraints are approximated by a linear equation, as in [9]. The voltage in a node is expressed as:

$$
\begin{aligned}
V_{h, p h}= & V_{n}+\sum_{i=1}^{n} P_{i} \cdot\left(P h_{i, 1} \cdot \mu_{h 1, i}+P h_{i, 2} \cdot \mu_{h 2, i}\right. \\
& \left.+P h_{i, 3} \cdot \mu_{h 3, i}\right)
\end{aligned}
$$

Where:

- $\quad V_{h, p h}$ is the voltage at node $h$ and phase $p h$

- $V_{n}$ is the grid nominal voltage, herein $230 \mathrm{~V}$

- $\quad P_{i}$ is the active power consumed by the load at node $\mathrm{i}$

- $\quad P h_{i, p h}$ is the phase vector for node $i$, containing 1 or 0

- $\quad \mu_{h 1, i}$ represents the influence of the power of load $i$ (if connected in phase 1) over the load voltage in node $h$ at phase $p h$

\section{PHASE BALANCING}

When three-phase PV inverters are replaced by three separately controlled single phase inverters with common DC-bus more generated power can be injected in the overloaded phases compared to the other phases.

To control the power flow of a balancing inverter, only the absolute value of the local voltage measurements are used. Phases with a lower voltage have a higher active power consumption due to the voltage drop over the mainly resistive distribution cables. Therefore it is interesting to inject more generated power in the overloaded phases. This is translated into the following droop relationship:

$$
\begin{gathered}
P_{U}-P_{V}=\gamma\left(V_{U}-V_{V}\right) \\
P_{U}-P_{W}=\gamma\left(V_{U}-V_{W}\right) \\
P_{V}-P_{W}=\gamma\left(V_{V}-V_{W}\right) \\
P_{U}+P_{V}+P_{W}=P_{P V}
\end{gathered}
$$

$\mathrm{P}_{\mathrm{U}, \mathrm{V}, \mathrm{W}}$ is the active power absorbed by the respective phase $\mathrm{U}, \mathrm{V}$ or $\mathrm{W} . \mathrm{P}_{\mathrm{PV}}$ is the power generated by the $\mathrm{PV}$ panel and $\mathrm{V}_{\mathrm{U}, \mathrm{V}, \mathrm{W}}$ is the absolute value of the voltage of the respective phase $\mathrm{U}, \mathrm{V}$ or $\mathrm{W} . \gamma$ is a parameter controlling the inter phase power delivery.

Equation (2) can be rewritten as:

$$
\begin{aligned}
& P_{U}=\frac{P_{P V}+\gamma\left(V_{U}-V_{V}\right)+\gamma\left(V_{U}-V_{W}\right)}{3} \\
& P_{V}=P_{U}-\gamma\left(V_{U}-V_{V}\right) \\
& P_{W}=P_{U}-\gamma\left(V_{U}-V_{W}\right)
\end{aligned}
$$

This distribution of power across the different phases is presented in Figure 1 for a $\gamma$ equal to -250 , meaning that 1 Volt of difference between two phases results in $250 \mathrm{~W}$ being more injected in the phase with the lowest voltage. Even at night full inverter capacity is used to transfer power from high loaded phases to low loaded phases. At night the power can be extracted from low loaded phases and injected into heavily loaded phases, with a net exchanged power with the grid $\mathrm{P}_{\mathrm{PV}}$ equal to zero. Inverter constraints are taken into account by projecting the values obtained by the droop relationship onto the feasible set of the inverter.

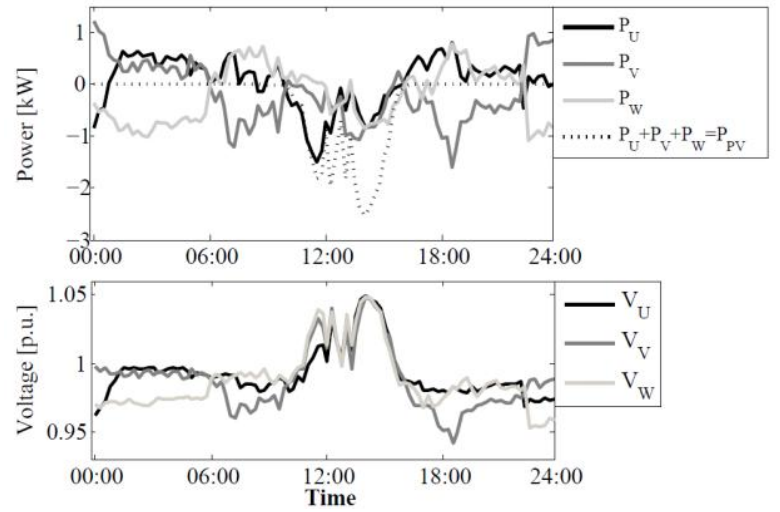

Figure 1: Power of the balancing inverter as a function of the grid voltage

\section{RESULTS}

All simulations are performed on a typical three-phase four-wire Belgian 230V low-voltage city-feeder provided by the DSO, presented in Figure 2. More information about the topology can be found in [10]. A database of statistically representative residential load profiles was available to perform load flow simulations. Two cases will be simulated and compared. In the first simulation $50 \%$ of the households connected to phase $\mathrm{U}$ are equipped with a single-phase PV generator and two households have a PV generator with three-phase connection. This results in unbalanced grid conditions, especially at moments of high PV production. This simulation will be called the highly unbalanced grid. In the second simulation $50 \%$ of all the houses have a PV 
generator, randomly distributed across the three phases and the same two houses are equipped with a three phase PV production unit. This simulation will be called the normal balanced grid.
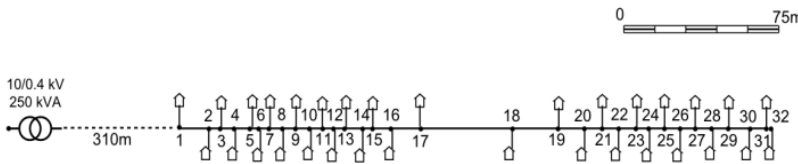

\section{Figure 2: Simulated distribution grid}

Within this study, the number of phase changes has been limited to five. As well, the changes are considered to be permanent during one year, so that the unbalance results with and without phase change can be compared. Figure 3 shows the voltage unbalance after some phase changes, starting from the initial configuration. Herein, the daily maximum unbalance in the grid is plotted for few days. The figure illustrates that with only few phase changes, a considerable unbalance improvement can be achieved.

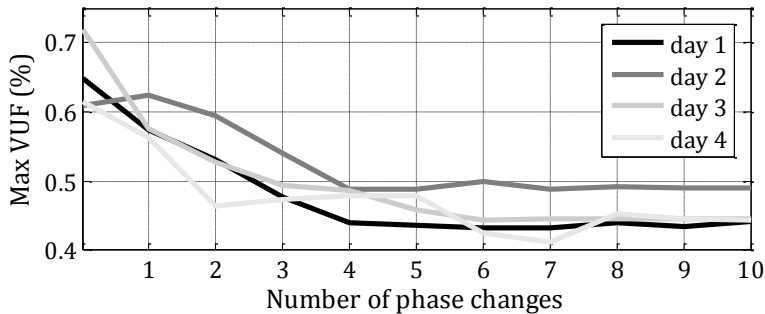

Figure 3: Voltage Unbalance Factor after different phase changes

To test the proposed balancing inverter control, the two standard three-phase inverters are replaced by three single phase inverters with common DC-bus that balance the grid with a droop constant $\gamma$ equal to $250 \frac{W}{V}$.

Figure 4 and Figure 5 compare the weekly maximum and minimum voltages after applying the phase changes or replacing two standard 3-phase inverters with balancing inverters for both the highly unbalanced grid as well as the normal balanced grid. $\mathrm{V}_{\text {,original }}$ are the voltages without any measures. $\mathrm{V}_{\text {.ph-bal }}$ are the voltages in case of phase balancing and $\mathrm{V}_{\text {,ph-switch }}$ are the voltages in case of phase switching.

As a result of the phase switching, the maximum voltage in average decreases. As the phase of connection is kept constant during the year, the minimum voltage at certain time instances become worse. Applying phase switching is mainly interesting when PV-inverters are unequally distributed across the phases. By switching some of them to other phases, the maximum occurring voltage decreases significantly. Phase switching is a labourintensive task as a technical crew is required to switch the phases or a phase switching device which can be controlled from a distance has to be installed.
In contrast to the phase switching, the phase balancing improves both minimum and maximum voltage at all time. The balancing inverter adapts to changing conditions. It is not labour-intensive but comes at the cost of extra hardware requirements. The highest improvement of grid conditions with the proposed inverter control occurs at the evening. At the evening, when no PV power is generated and high peak loads occur, full inverter capacity can be used to balance the grid and therefore mainly the minimum voltage occurring in the grid increases.

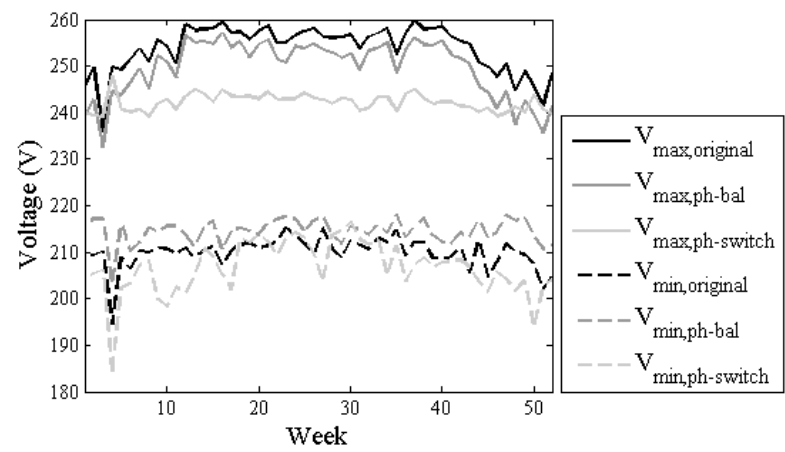

Figure 4: Maximum and minimum voltage in a highly unbalanced grid with phase balancing and phase switching

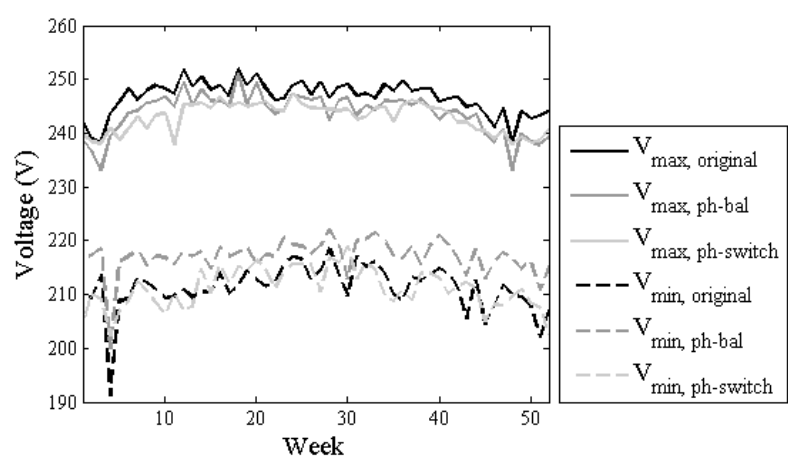

Figure 5: Maximum and minimum voltage in a normal loaded grid with phase balancing and phase switching

The maximum $95^{\text {th }}$ VUF percentile during a week is plotted in Figure 6 for the highly unbalanced grid and in Figure 7 for the normal loaded grid. It is clear from these figures that phase switching improves the unbalance in case the PV is unequally distributed across the phases, but leads to no improvement when the PV is spread across all the phases. Phase balancing improves the unbalance in both cases. The improvements of the balancing inverter depend on the droop constant $\gamma$, the number of balancing inverters and their location.

Balanced radial distribution grids can supply more power to loads. Therefore the proposed system can help with the integration of electric vehicles in the grid as well. 


\section{CONCLUSION}

Switching the phase of connection of single phase customers is shown to be effective in case the distribution grid has an unequal distribution across the phases of the PV installations. The changes are considered to be permanent during one year and therefore it is impossible to improve the balance in the grid continuously during this period, because customer behavior varies frequent. Balancing the grid by three single phase inverters with common DC-bus significantly improves grid conditions at all time and can further improve grid conditions, even in case loads and PV generators are already equally distributed across the phases. The phase balancing does not suffer from the continuously changing behaviour from customers. The control depends on the local voltage measurements and therefore can adapt to changing conditions. No communication is required.

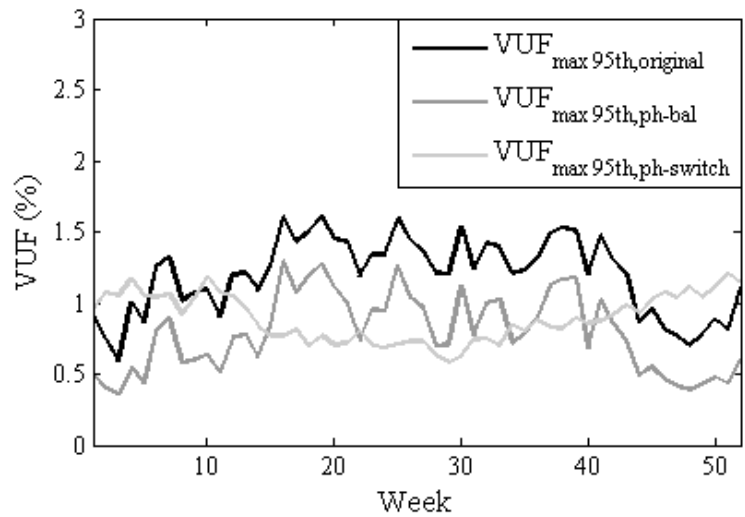

Figure 6: Yearly Voltage Unbalance Factor with phase balancing and switching for a highly unbalanced grid

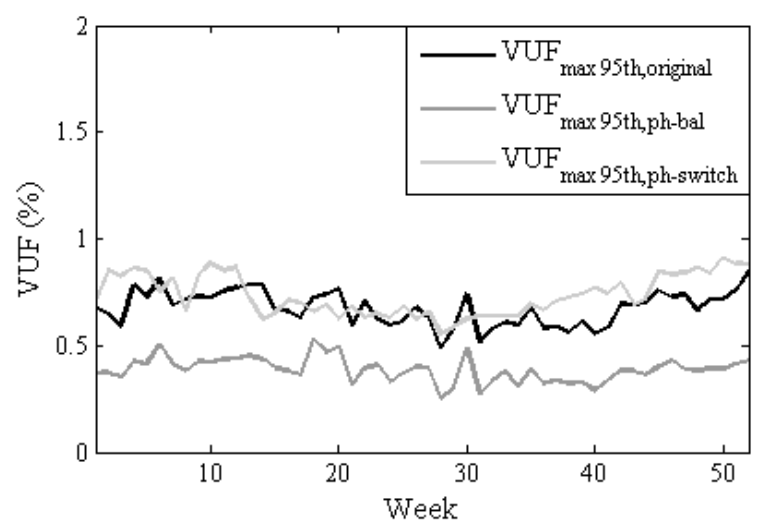

Figure 7: Yearly Voltage Unbalance Factor with phase balancing and switching in a normal loaded grid

\section{ACKNOWLEDGEMENT}

The work is supported by the Flemish Government through the LINEAR project (IWT/090800) organized by the Institute for Science and Technology (IWT). S.
Weckx has a PhD fellowship of the Research Foundation - Flanders - VITO (FWO - VITO).

\section{REFERENCES}

[1] Y. Tan, D. Kirschen, N. Jenkins, 2003, "Impact of a large penetration of photovoltaic generation on the power system," CIRED 17th International conference on Electricity Distribution

[2] F. Shahnia, R. Majumder, A. Ghosh, G. Ledwich et F. Zare, 2010 "Sensitivity analysis of voltage imbalance in distribution networks with rooftop PVs," IEEE Power and Energy Society General Meeting, p 1-8.

[3] S. Conti, A. Greco, N. Messina and S. Raiti, 2006 "Local voltage regulation in LV distribution networks with PV distributed generation," International Symposium on Power Electronics, Electrical Drives, Automation and Motion, 2006. SPEEDAM 2006., pp 519 - 524 .

[4] B. Meersman, B. Renders, L. Degroote, T. Vandoorn and L. Vandevelde, 2011 "Three-phase inverter-connected DG-units and voltage unbalance," Electric Power Systems Research, vol. $81, \mathrm{n}^{\circ} 14$, pp. 899-906.

[5] F. Wang, J. Duarte and M. Hendrix, 2008 "Control of grid-interfacing inverters with integrated voltage unbalance correction," Power Electronics Specialists Conference, 2008. PESC 2008. IEEE, pp 310 -316.

[6] Synergrid, 2012 Specifieke Technische Voorschriften Voor Decentrale Productie-Installaties die in Parallel Werken met het Distributienet, Synergrid, .

[7] W. Siti, A. Jimoh and Nicolae. D., 2011 "Distribution network phase load balancing as a combinatorial optimization problem using fuzzy logic and newtonraphson," Electric Power Systems Research, vol. 81, $\mathrm{n}^{\circ} .5$, pp. $1079-1087$.

[8] C. Cheng et D. Shirmohammadi, 1995 "A threephase power flow method for real-time distribution system analysis," IEEE Transactions on Power Systems, vol. 10, pp. 671 - 679

[9] P. Richardson, D. Flynn and A. Keane, 2012 "Optimal Charging of Electric Vehicles in LowVoltage Distribution Systems," IEEE Transactions on Power Systems, vol. 27, $\mathrm{n}^{\circ} 11$, pp. 268-279

[10] C. Gonzalez, J. Geuns, S. Weckx, T. Wijnhoven, P. Vingerhoets and J. Driesen, 2012 "LV Distribution Network Feeders in Belgium and Power Quality Issues due to Increasing PV Penetration Levels," IEEE Innovative smart grid technologies, pp 1-8 